\title{
Punção Aspirativa de Nódulos da Tiroide: Vale a Pena Repetir?
}

\section{Fine-needle Aspiration of Thyroid Nodules: Is it Worth Repeating?}

\author{
Vera FERNANDES $\triangle^{1,2,3}$, Tânia PEREIRA ${ }^{4}$, Catarina ELOY ${ }^{4,5}$ \\ Acta Med Port 2017 Jun;30(6):472-478 - https://doi.org/10.20344/amp.8215
}

\section{RESUMO}

Introdução: A punção aspirativa por agulha fina tem um papel relevante na avaliação do risco de malignidade de nódulos da tiroide. Persistem dúvidas quanto ao valor da repetição da punção aspirativa por agulha fina em nódulos com resultado benigno. Este trabalho pretende avaliar a concordância de resultados em punções aspirativas por agulha fina consecutivas e estudar a pertinência da repetição nos resultados benignos.

Material e Métodos: Estudo retrospetivo dos resultados das 4800 punções aspirativas por agulha fina da tiroide realizadas no Instituto de Patologia e Imunologia Molecular da Universidade do Porto entre 1 de janeiro de 2014 e 2 de maio de 2016. Da amostra inicial, selecionaram-se as punções aspirativas por agulha fina repetidas no mesmo nódulo.

Resultados: O resultado da primeira punção aspirativa por agulha fina dos 309 nódulos submetidos a reavaliação foi não diagnóstico em 103 (33,3\%), benigno em 120 (38,8\%) e atipia/lesão folicular de significado indeterminado em 86 (27,8\%). A concordância entre a primeira e segunda punção aspirativa por agulha fina foi significativamente mais elevada nos casos com resultado inicial benigno (benigno: $85,8 \%$, não diagnóstico: $27,2 \%$ e atipia/lesão folicular de significado indeterminado: $17,4 \%, p<0,005$ ). Os motivos de repetição da punção aspirativa por agulha fina em resultados inicialmente benignos $(n=78)$ foram a sugestão de repetição em 58 , crescimento do nódulo em 17 e características ecográficas suspeitas de malignidade em três.

Discussão: A repetição da punção aspirativa por agulha fina em nódulos com resultado inicial não diagnóstico e atipia/lesão folicular de significado indeterminado alterou o diagnóstico inicial numa proporção significativa de doentes, modificando a sua orientação terapêutica. A elevada concordância de resultados em casos inicialmente benignos torna a repetição da punção aspirativa por agulha fina não custo-efetiva na maioria dos casos.

Conclusão: A repetição da punção aspirativa por agulha fina deve ser realizada quando o resultado citológico inicial é não diagnóstico ou atipia/lesão folicular de significado indeterminado.

Palavras-chave: Biópsia por Agulha Fina; Nódulo da Tiroide

\section{ABSTRACT}

Introduction: The fine-needle aspiration has a significant role in assessing the malignancy risk of thyroid nodules. There is uncertainty regarding the value of repeat fine-needle aspiration in benign nodules. This study aims to evaluate the concordance of results in consecutive fine-needle aspiration and to study the relevance of repetition in benign results.

Material and Methods: Retrospective study of the 4800 thyroid nodules fine-needle aspiration held in Instituto de Patologia e Imunologia Molecular da Universidade do Porto between January 1, 2014 and May 2, 2016. Of the initial sample, we selected the repeated fine-needle aspiration on the same nodule.

Results: The first fine-needle aspiration result of the 309 nodules underwent revaluation was non-diagnostic in 103 (33.3\%), benign in $120(38.8 \%)$ and atypia/follicular lesion of undetermined significance in $86(27.8 \%)$. The agreement between the first and second fine-needle aspiration was significantly higher in cases with an initial benign result (benign: $85.8 \%$, non-diagnostic: $27.2 \%$ and atypia/ follicular lesion of undetermined significance: $17.4 \%, p<0.005)$. The fine-needle aspiration repeating motifs in initially benign nodules $(n=78)$ were repetition suggestion in 58 , nodule growth in 17 and suspicious ultrasonographic features in 3.

Discussion: The fine-needle aspiration repetition in nodules with initial non-diagnostic and atypia/follicular lesion of undetermined significance result changed the initial diagnosis in a significant proportion of patients, modifying their therapeutic approach. The high concordance of results in initially benign nodules makes fine-needle aspiration repetition not cost-effective in most cases.

Conclusion: The fine-needle aspiration should be repeated when the initial cytology result is non-diagnostic or atypia/follicular lesion of undetermined significance.

Keywords: Biopsy, Fine-Needle; Thyroid Nodule

\section{INTRODUÇÃO}

A patologia nodular da tiroide é muito frequente, apresentando uma prevalência de 50 a $60 \%$ na população geral. ${ }^{1}$ Em casos de nódulos da tiroide, o desafio é selecionar os nódulos malignos para tratamento cirúrgico. A punção aspirativa por agulha fina (PAAF) destaca-se neste contex- to pelo seu papel na identificação de nódulos com maior risco de malignidade. ${ }^{2,3}$

APAAF é um meio complementar de diagnóstico de execução relativamente simples e com poucas complicações associadas, ${ }^{4}$ que apresenta um valor preditivo negativo

1. Serviço de Endocrinologia. Hospital de Braga. Braga. Portugal.

2. ICVS - Instituto de Investigação em Ciências da Vida e Saúde. Escola de Ciências da Saúde. Universidade do Minho. Braga. Portugal.

3. ICVS/3Bs - PT Government Associate Laboratory. Braga/Guimarães. Portugal.

4. IPATIMUP Diagnósticos. Instituto de Patologia e Imunologia Molecular. Universidade do Porto. Porto. Portugal.

5. Departamento de Patologia. Faculdade de Medicina. Universidade do Porto. Porto. Portugal.

$\triangle$ Autor correspondente: Vera Fernandes. vera.fernandes20@gmail.com

Recebido: 02 de março de 2016 - Aceite: 18 de novembro de 2016 | Copyright @ Ordem dos Médicos 2017 
(VPN) de 95\% e um valor preditivo positivo (VPP) de 97 a $99 \% .^{5}$

De acordo com as recomendações internacionais, a indicação para realização de PAAF da tiroide relaciona-se com as características ecográficas e dimensão do nódulo. ${ }^{1,6}$ Quando os resultados da PAAF estão associados a um elevado risco de malignidade não há dúvida na orientação cirúrgica dos casos, porém, nos restantes resultados, questiona-se a utilidade da repetição da PAAF. Recomenda-se repetir a PAAF em casos com resultado não diagnóstico ou de atipia de significado indeterminado/lesão folicular de significado indeterminado (AUS/FLUS do acrónimo em inglês), segundo a classificação de Bethesda. ${ }^{3,7}$ A repetição da PAAF em casos com resultado benigno é controversa. Alguns estudos apoiam a repetição da PAAF argumentando uma diminuição da taxa de falsos negativos e, portanto, uma orientação cirúrgica precoce em casos malignos. ${ }^{8-10}$ Outros autores defendem a não repetição da PAAF atendendo à baixa taxa de falsos negativos no exame inicial e à ausência de impacto prognóstico com um eventual atraso no diagnóstico. ${ }^{11}$

Os principais objetivos deste trabalho consistiram em avaliar a concordância de resultados em PAAFs consecutivas da tiroide e verificar a pertinência das repetições de PAAFs inicialmente classificadas como benignas.

\section{MATERIAL E MÉTODOS}

Efetuou-se um estudo retrospetivo dos resultados das PAAFs da tiroide realizadas com controlo ecográfico, de 01 de janeiro de 2014 a 02 de maio de 2016, no Instituto de Patologia e Imunologia Molecular da Universidade do Porto (IPATIMUP).

As PAAFs dos nódulos da tiroide e a interpretação da citologia, salvo raras exceções, foram efetuadas pelo mesmo patologista. Todos os resultados das PAAFs foram relatados em conformidade com o sistema de Bethesda. ${ }^{3}$ De acordo com o mesmo sistema, as biopsias não diagnósticas são aquelas que não cumprem os critérios quantitativos ou qualitativos estabelecidos para uma citologia adequada, estando preconizada a presença de pelo menos seis grupos de células foliculares, cada grupo com pelo menos dez células epiteliais bem preservadas. ${ }^{3}$ Amostras com quantidade e qualidade celular no limite do aceitável foram classificadas como benignas, com sugestão de repetição da biopsia num período não inferior a três meses; daqui em diante considerado um subtipo de resultado benigno e designadas por benigno - $\mathrm{R}$.

De uma amostra inicial de 4800 PAAFs realizadas no período em estudo, selecionaram-se as PAAFs repetidas num mesmo doente e consultaram-se todos os relatórios correspondentes. Os critérios de exclusão foram: segunda PAAF em nódulo distinto do primeiro e ausência de informação quanto ao nódulo estudado (na primeira, segunda ou em ambas as punções). Apenas oito nódulos (2,6\%) foram avaliados três vezes. Por se considerar este grupo de oito nódulos pouco representativo, não haverá referência à terceira avaliação do nódulo.
Os dados foram colhidos com recurso ao programa informático SISPAT ${ }^{\circledR}$ e exportados para o programa Microsoft Office Excel ${ }^{\circledR}$ 2011. Para a análise estatística recorreu-se ao programa estatístico IBM ${ }^{\circledR}$ SPSS $^{\circledR}$ Statistics v. 22. Os dados relativos às variáveis categóricas foram sumariados em tabelas de frequências. Relativamente às variáveis contínuas, avaliou-se a normalidade da distribuição e descreveram-se na forma de média \pm desvio padrão ou mediana e amplitude interquartil (AIQ). Na análise inferencial recorreu-se ao teste qui-quadrado de Pearson para avaliar a associação entre variáveis qualitativas. $O$ teste $t$-student e one-way ANOVA foram utilizados para a comparação de médias entre variáveis com distribuição normal e os testes não-paramétricos Mann-Whitney e Kruskal-Wallis foram usados para o estudo de variáveis com uma distribuição não normal.

Considerou-se existir significância estatística para valores de $p<0,05$ e tendência estatisticamente significativa se $0,05 \leq p<0,1$. Durante todas as fases de elaboração do estudo foram respeitados os princípios éticos e deontológicos referentes à boa prática de recolha e análise estatística dos dados.

\section{RESULTADOS}

Os resultados das 4800 PAAFs da tiroide realizadas no IPATIMUP no período em estudo encontram-se distribuídos na Fig. 1. Da amostra inicial, 638 correspondiam a PAAFs repetidas no mesmo nódulo da tiroide. Após a aplicação dos critérios de exclusão, obteve-se uma amostra final constituída por 309 nódulos, correspondentes a 291 doentes.

Os 309 nódulos analisados foram detetados em indivíduos com média de idade de $54 \pm 14$ anos, variando de 18 a 87 anos, sendo a maioria do género feminino (84,5\%). A mediana do período entre a primeira e segunda PAAF foi de seis meses (AIQ: P25: 4 - P75: 9). Relativamente à localização dos nódulos, 3,6\% encontravam-se no istmo, $52,1 \%$ no lobo direito e $44,3 \%$ no lobo esquerdo. A mediana da dimensão dos nódulos, aquando da primeira PAAF, foi de $15 \mathrm{~mm}$ (12 - 19), variando entre 5,8 e $50 \mathrm{~mm}$, e aquando da segunda PAAF foi de $16 \mathrm{~mm}(12-21,8)$, variando entre 7 e $85 \mathrm{~mm}$.

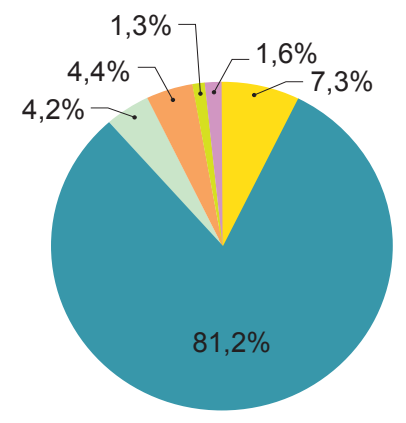
๑ I Não diagnóstico
๑ II Benigno
๑ III AUS/FLUS
口 IV Tumor folicular
$\square$ V Suspeito de malignidade
$\square$ VI Maligno

Figura 1 - Distribuição das 4800 PAAFs de acordo com resultado citológico, segundo classificação de Bethesda

AUS/FLUS: Atypia of Undetermined Significance or Follicular Lesion of Undetermined Significance 


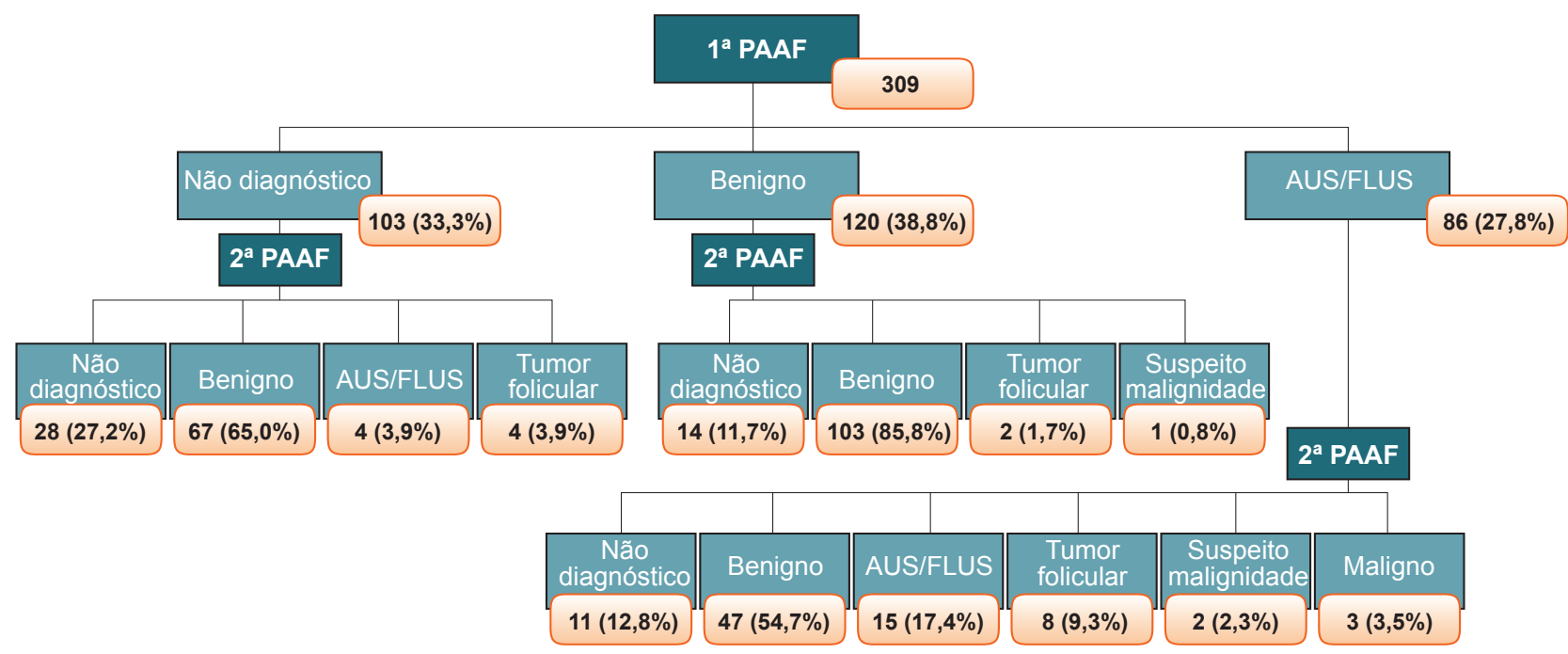

Figura 2 - Distribuição dos 309 casos com repetição de PAAF de acordo com os resultados citológicos obtidos, segundo classificação de Bethesda

AUS/FLUS: Atypia of Undetermined Significance or Follicular Lesion of Undetermined Significance

Os 309 nódulos submetidos a reavaliação citológica numa segunda PAAF foram classificados na primeira PAAF numa de três categorias diagnósticas da classificação de Bethesda: 103 (33,3\%) apresentaram resultado não diagnóstico, 120 (38,8\%) benigno e 86 (27,8\%) AUS/FLUS (Fig. 2).

Os dois casos com primeira PAAF com resultado benigno e segunda com resultado de tumor folicular, correspondiam ambos a nódulos do istmo, um de $30 \mathrm{~mm}$ e resultado de hiperplasia de células foliculares, e o outro de $43 \mathrm{~mm}$ e com crescimento de $7 \mathrm{~mm}$ em 11 meses. O caso de primeira PAAF com resultado benigno e segunda com resultado suspeito de malignidade apresentava quantidade celular no limite do aceitável e tinha no relatório uma recomendação para a repetição, que aconteceu seis meses depois.

Os nódulos inicialmente classificados como AUS/FLUS, foram reclassificados pelas seis categorias da classificação de Bethesda na segunda PAAF, sendo a única classe diagnóstica com resultados de malignidade na reavaliação.
A análise das variáveis demográficas, características dos nódulos e concordância dos resultados entre PAAFs, por categoria diagnóstica da primeira PAAF, está sumariada na Tabela 1. No que diz respeito às características demográficas e localização do nódulo, não foi detetada diferença estatisticamente significativa entre os grupos com os diferentes resultados. Foi observada uma concordância significativamente mais elevada entre a primeira e segunda PAAF quando o resultado inicial foi benigno $(85,8 \%)$, em comparação com concordância entre PAAFs quando o resultado inicial foi não diagnóstico ou AUS/FLUS $(27,2 \%$ e $17,4 \%$, respetivamente) $(p<0,005)$. A elevada concordância entre resultados benignos ascende aos $95,4 \%$ se eliminadas as amostras classificadas como benigno- $R$ (concordância em casos benigno-R: $74,5 \%$ ) (Tabela 2).

Os motivos de repetição da PAAF em resultados benignos puderam ser avaliados em 78 PAAFs $(32,1 \%$ explícitos na informação clínica e $67,9 \%$ inferidos pelo contexto clínico) e foram os seguintes: sugestão de repetição de

Tabela 1 - Variáveis demográficas, características dos nódulos e resultados de segunda PAAF, por categoria diagnóstica obtida na primeira PAAF

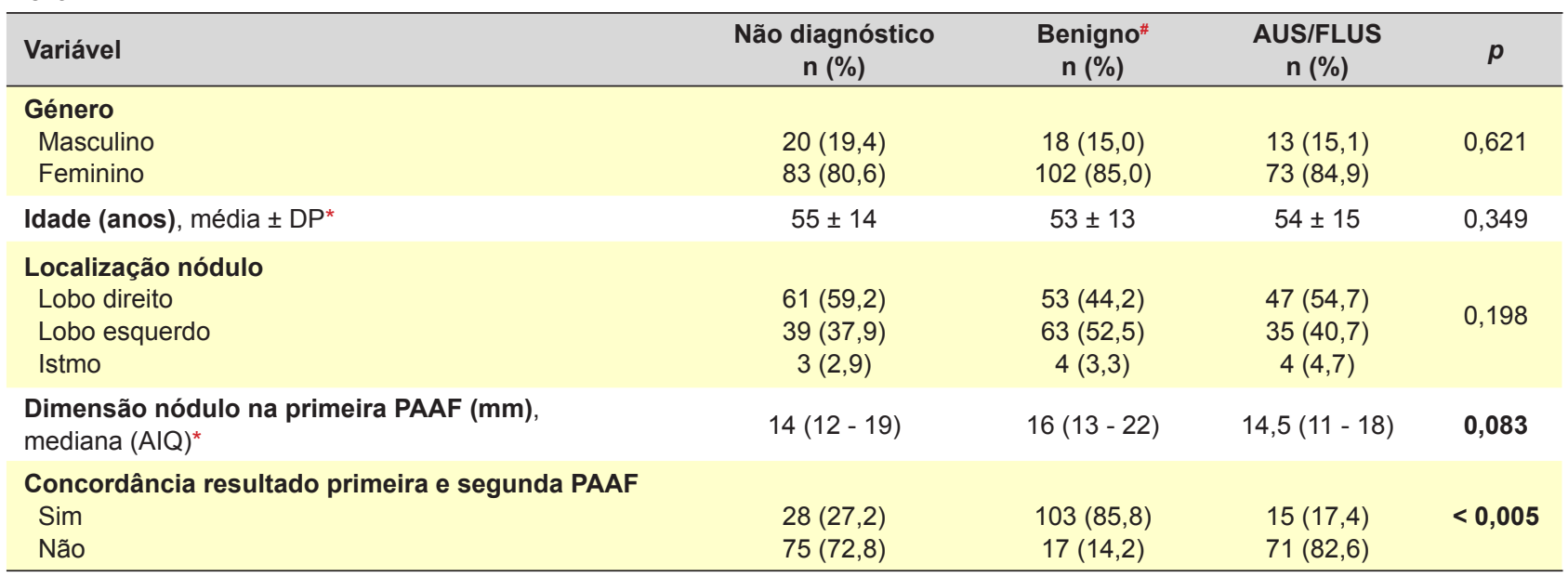

${ }^{*}$ Resultados apresentados na forma de n(\%), a não ser que especificado. \# Inclui benigno-R. DP: Desvio padrão; AIQ: Amplitude interquartil; PAAF: Punção aspirativa por agulha fina 
Tabela 2 - Estudo condordância de resultados da segunda PAAF de acordo com resultado de primeira PAAF não diagnóstico, benigno-R ou benigno (casos que não incluem os benignos-R)

\begin{tabular}{|c|c|c|c|c|c|}
\hline Segunda PAAF & Primeira PAAF & $\begin{array}{c}\text { Não diagnóstico } \\
\mathrm{n}(\%)\end{array}$ & $\begin{array}{c}\text { Benigno-R } \\
\text { n (\%) }\end{array}$ & $\begin{array}{c}\text { Benigno } \\
\mathrm{n}(\%)\end{array}$ & $p$ \\
\hline \multicolumn{6}{|l|}{ Resultado } \\
\hline Não diagnóstico & & $28(27,2)$ & $13(23,6)$ & $1(1,5)$ & \multirow{5}{*}{ ND } \\
\hline Benigno & & $67(65)$ & $41(74,5)$ & $62(95,4)$ & \\
\hline AUS/FLUS & & $4(3,9)$ & 0 & 0 & \\
\hline Tumor folicular & & $4(3,9)$ & 0 & $2(3,1)$ & \\
\hline Suspeito de malignidade & & 0 & $1(1,8)$ & 0 & \\
\hline
\end{tabular}

ND: Não determinável, porque não estão reunidos os pressupostos necessários à utilização do teste estatístico qui-quadrado

PAAF em relatório prévio em 58 nódulos (quando resultado benigno - $\mathrm{R}$ ou com hiperplasia de células foliculares), crescimento do nódulo em 17 nódulos (6 com informação explícita e 11 sem essa informação mas com aumento de dimensão entre PAAFs) e características ecográficas suspeitas de malignidade em três nódulos.

Dos 15 nódulos com resultado consistentemente AUS/ FLUS na primeira e segunda PAAF, obtiveram-se dados do seguimento clínico em 12 (80\%). Desses 12 casos, cinco $(41,7 \%)$ foram orientados para tratamento cirúrgico, com diagnóstico histológico de malignidade em dois casos $(40 \%$ de risco de malignidade), benignidade em dois casos e houve um caso de diagnóstico de benignidade com microcarcinoma papilar incidental não amostrado na PAAF.

\section{Dimensão dos nódulos}

A maior dimensão dos nódulos da tiroide aquando da primeira PAAF e da segunda PAAF encontra-se distribuída por categoria diagnóstica nas Fig.s 3 e 4, respetivamente.

Dos 103 nódulos com resultado não diagnóstico na primeira PAAF, apenas 79 tinham informação quanto à dimensão do nódulo. Destes 79 nódulos, cinco $(6,3 \%)$ apresentavam dimensão inferior a $10 \mathrm{~mm}$ e $41(51,9 \%)$ inferior a 15 $\mathrm{mm}$. Nos casos que mantiveram resultado não diagnóstico na segunda PAAF $(n=20)$, um ( $5 \%)$ apresentava dimensão inferior a $10 \mathrm{~mm}$ e $15(75 \%)$ inferior a $15 \mathrm{~mm}$.

Os nódulos com resultado AUS/FLUS e não diagnóstico na primeira PAAF foram significativamente $(p=0,039)$ e tendencialmente $(p=0,08)$ menores que os nódulos

$p=0,651$

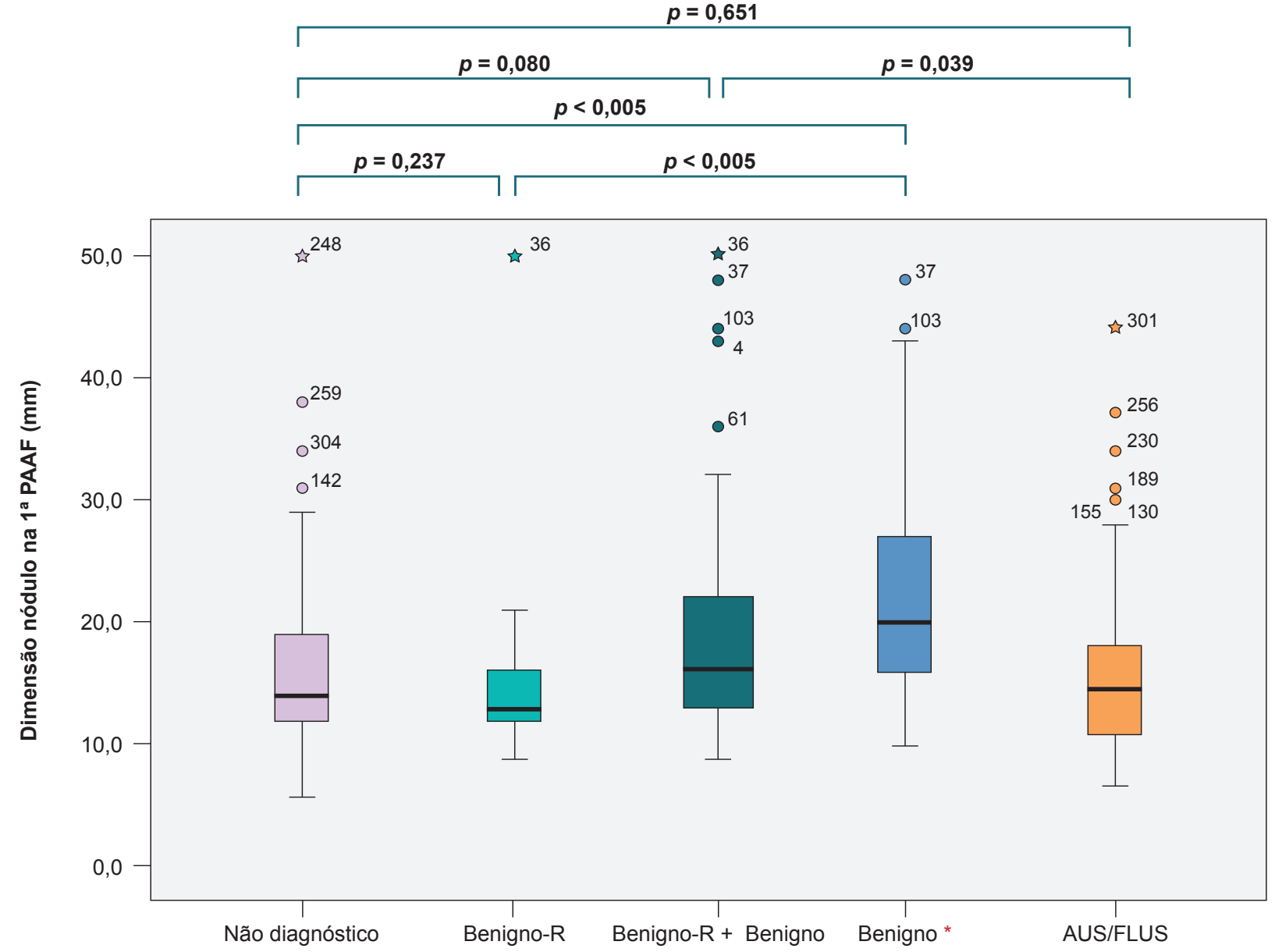

Classificação citologias (sistema de Bethesda) na $1^{\text {a }}$ PAAF

Figura 3 - Dimensão dos nódulos da citologia inicial, segundo classificação de Bethesda

AUS/FLUS: Atypia of Undetermined Significance or Follicular Lesion of Undetermined Significance. * Não inclui benigno-R. 
benignos (incluindo benigno-R), respetivamente (Fig. 3). Os nódulos benignos- $\mathrm{R}$ tinham tamanho semelhante àqueles designados como não-diagnósticos ( $p=0,237)$.

Aquando da segunda PAAF, os nódulos com resultado não diagnóstico eram menores que os demais (não diagnóstico: $12 \mathrm{~mm}$ versus benigno: $17 \mathrm{~mm}$ versus AUS/FLUS $16 \mathrm{~mm}$ versus tumor folicular: $25 \mathrm{~mm}$ versus suspeito de malignidade: $24 \mathrm{~mm}, p<0,005)$ (Fig. 4).

\section{Período entre punções}

O período entre a primeira e a segunda PAAF foi significativamente mais prolongado no grupo com diagnóstico inicial benigno. O grupo de nódulos com resultado benigno - $\mathrm{R}$ foi submetido a segunda PAAF significativamente mais tarde que o grupo com resultado não diagnóstico na primeira PAAF (não diagnóstico: cinco meses vs benigno-R: sete meses, $p<0,005)$ e significativamente mais cedo que os restantes benignos (sete meses versus 14 meses, respetivamente, $p<0,005)$. Não se verificaram diferenças estatisticamente significativas entre o grupo com resultado inicial não diagnóstico e AUS/FLUS (não diagnóstico: cinco meses versus benigno: 10 meses versus AUS/FLUS: quatro meses, $p<0,005)$.

\section{DISCUSSÃO}

A série de PAAFs da tiroide estudada contempla casos com uma distribuição de resultados adequada segundo a classificação de Bethesda ${ }^{3}$ e inclui repetições de PAAF que foram efetuadas apenas em nódulos com resultados não diagnóstico, benigno e AUS/FLUS.

Os dados obtidos neste estudo permitem concluir que há benefício em repetir a PAAF em nódulos da tiroide com resultado inicial não diagnóstico e que a amostra obtida na repetição será adequada em $73 \%$ dos nódulos. A repetição da PAAF num nódulo com resultado citológico prévio não diagnóstico teve um diagnóstico de benignidade em $65 \%$ dos casos, permitindo manter estes doentes em vigilância clínica e evitando o sobre-tratamento. Uma das razões que parece estar subjacente ao resultado não diagnóstico na primeira PAAF desta série é o tamanho dos nódulos. De facto, os nódulos com resultados não diagnóstico na primeira PAAF foram significativamente mais pequenos que os nódulos com resultados benignos (exceto benigno-R) na primeira PAAF e segunda PAAF. ${ }^{12}$ Assim sendo, será de ponderar a vigilância ecográfica destes nódulos pequenos até se observar crescimento do nódulo, aumentando as possibilidades de uma melhor amostragem.

Relativamente à repetição da PAAF em nódulos com resultado benigno, apesar da controvérsia existente, os dados deste trabalho apoiam a não repetição da PAAF na maioria dos nódulos. Cerca de $86 \%$ dos nódulos com PAAF revelando resultado benigno permanece com resultado benigno na segunda PAAF e a proporção de falsos negativos aparente (ausência de resultados histológicos) é de apenas 2,5\% (três casos). Assim, segundo o estudo nesta série, a repetição da PAAF nos nódulos com resultado benigno não é custo-efetiva e não deve ser realizada, a não ser que os nódulos sejam grandes, cresçam ou

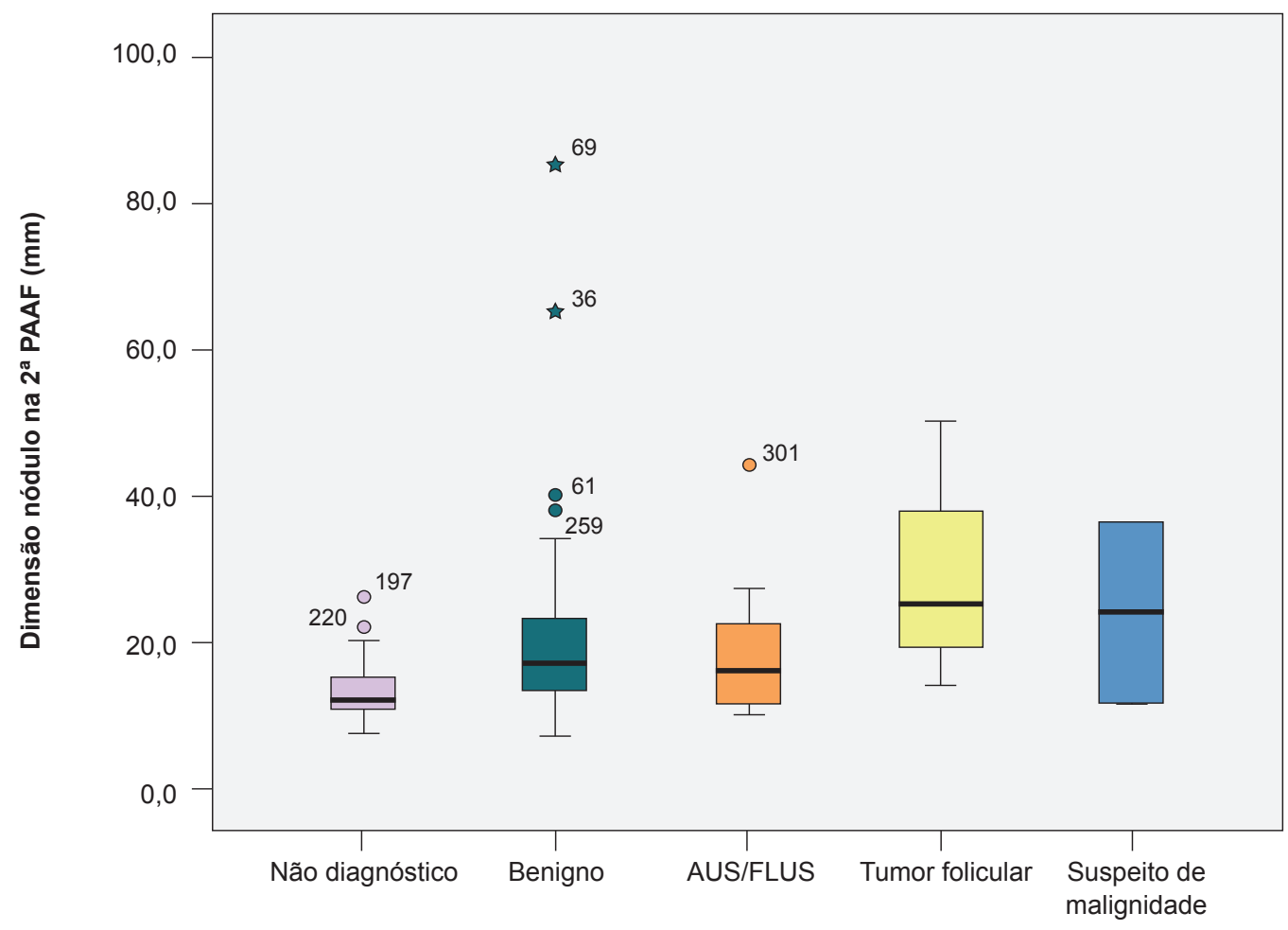

Classificação citologias (sistema de Bethesda) na $2^{\text {a }}$ PAAF

Figura 4 - Dimensão dos nódulos aquando da reavaliação citológica, segundo classificação de Bethesda AUS/FLUS: Atypia of Undetermined Significance or Follicular Lesion of Undetermined Significance. ${ }^{*}$ Inclui benigno-R. 
apresentem hiperplasia de células foliculares. Os resultados obtidos mostram que os nódulos com resultado benigno-R são nódulos benignos com material escasso condicionado, provavelmente, pela sua reduzida dimensão. Estes nódulos mostraram ser significativamente mais pequenos que os restantes benignos e os resultados da segunda PAAF aproximam-se mais daqueles do grupo não diagnóstico do que benigno. Verificou-se, ainda, que este resultado constituiu o principal motivo para repetição de PAAF em nódulos com resultado inicial benigno. Estes resultados sugerem que devemos ser exigentes no cumprimento dos critérios citológicos de adequação da amostra recomendados pelo Sistema de Bethesda ${ }^{3}$ e os nódulos benignos- $R$ devem seguir a mesma recomendação dos restantes nódulos com resultado não diagnóstico, justificando-se a recomendação para repetição da PAAF neste grupo, se forem preenchidos os critérios de tamanho do nódulo.

Os resultados das reavaliações por PAAF dos nódulos com diagnóstico de AUS/FLUS mostram que esta estratégia deve ser adotada, já que a repetição da PAAF é apropriada na maioria dos nódulos $(87,2 \%)$ e permite uma orientação terapêutica mais adequada e definitiva numa proporção significativa de doentes. Efetivamente, as reavaliações destes nódulos originaram 13 resultados $(15,1 \%)$ com risco de malignidade elevado (oito tumores foliculares, dois suspeitos de malignidade e três com critérios de malignidade), permitindo a orientação cirúrgica nestes casos. Além disso, mais de metade $(54,7 \%)$ das reavaliações por PAAF de nódulos com primeira PAAF com resultado AUS/ FLUS originaram um resultado benigno, evitando-se aqui o tratamento cirúrgico. O resultado inicial de AUS/FLUS em nódulos benignos poder-se-á dever a artefactos da preparação da amostra (secagem ao ar e coagulação da amostra), tiroidite linfocítica, tratamento prévio com iodo radioativo ou anti-tiroideus de síntese, e reparação celular em situações de degeneração cística e/ou hemorragia. ${ }^{3}$ Os achados morfológicos que conduzem a um resultado de AUS/FLUS devem ser reavaliados por um patologista experiente em contexto de segunda opinião, dado que esta medida pode obviar a necessidade de repetição da PAAF. Relativamente aos nódulos com resultado de PAAF persistentemente AUS/FLUS, os resultados mostram malignidade em $40 \%$ dos nódulos, pelo que se defende o tratamento cirúrgico nestes casos.

Uma proporção significativa dos nódulos estudados possuía pequenas dimensões (metade com diâmetro máximo de $15 \mathrm{~mm}$ na primeira PAAF). As recomendações para PAAF de nódulos da tiroide são cada vez mais conservadoras e o limite clássico de $1 \mathrm{~cm}$ foi substituído nas últimas recomendações da American Thyroid Association por 1,5 $\mathrm{cm}$. Os nódulos com diâmetro entre $1 \mathrm{~cm}$ e $1,4 \mathrm{~cm}$ devem ser submetidos a avaliação citológica apenas se possuírem caraterísticas imagiológicas suspeitas de malignidade ${ }^{1}$ e os nódulos infracentimétricos não têm indicação para essa avaliação por rotina atendendo a que os microcarcinomas papilares da tireoide $(<1 \mathrm{~cm})$ têm um comportamento in- dolente. ${ }^{1}$ Neste sentido, e atendendo à elevada realização de ecografias da tiroide na população em geral, destaca-se a importância do cumprimento das recomendações para avaliação por PAAF de nódulos da tiroide, ponderando o risco de se obterem resultados não diagnósticos e do sobre-tratamento (e suas complicações) que pode advir dos resultados obtidos.

Os resultados deste trabalho demonstram que o intervalo entre punções foi significativamente superior para os nódulos com resultado benigno na primeira PAAF, tal como previamente reportado, ${ }^{2}$ e provavelmente traduzem a menor preocupação do clínico com resultados associados a menor risco de malignidade.

Apesar deste estudo ter incluído um número substancial de PAAFs realizadas e interpretadas pelo mesmo patologista e reportar a realidade de um centro de referência nacional, apresenta algumas limitações que devem ser referidas. Trata-se de um estudo retrospetivo com falha de acesso a informação clínica completa na maioria dos casos, o que limitou o estudo do motivo de repetição de PAAF dos nódulos com resultado benigno, a correlação cito-histológica e o enquadramento com dados imagiológicos. Estudos futuros deverão contemplar a apreciação das características imagiológicas dos nódulos da tiroide que são os primeiros determinantes não só na decisão de avaliação citológica inicial, mas também na decisão de repetição de PAAF. ${ }^{1}$

\section{CONCLUSÃO}

Este estudo alerta para a importância da repetição da PAAF em doentes com resultado inicial não diagnóstico e AUS/FLUS, dado que altera a orientação terapêutica numa proporção significativa de doentes. Relativamente aos doentes com PAAF benigna, a repetição não parece ser custo-efetiva, exceto naqueles nódulos de maiores dimensões, com crescimento ou hiperplasia de células foliculares.

\section{PROTECÇÃO DE PESSOAS E ANIMAIS}

Os autores declaram que os procedimentos seguidos estavam de acordo com os regulamentos estabelecidos pelos responsáveis da Comissão de Investigação Clínica e Ética e de acordo com a Declaração de Helsínquia da Associação Médica Mundial.

\section{CONFIDENCIALIDADE DOS DADOS}

Os autores declaram ter seguido os protocolos do seu centro de trabalho acerca da publicação de dados.

\section{CONFLITOS DE INTERESSE}

Os autores declaram não terem qualquer conflito de interesse relativamente ao presente artigo.

\section{FONTES DE FINANCIAMENTO}

Os autores declaram não ter recebido subsídios ou bolsas para a elaboração do artigo. 


\section{REFERÊNCIAS}

1. Haugen BR, Alexander EK, Bible KC, Doherty GM, Mandel SJ, Nikiforov YE, et al. 2015 American Thyroid Association Management Guidelines for Adult Patients with Thyroid Nodules and Differentiated Thyroid Cancer: The American Thyroid Association Guidelines Task Force on Thyroid Nodules and Differentiated Thyroid Cancer. Thyroid. 2016;26:1133.

2. Graciano AJ, Chone CT, Fisher CA, Bublitz GS, Peixoto AJ. Repeated fine-needle aspiration cytology for the diagnosis and follow-up of thyroid nodules. Braz J Otorhinolaryngol. 2014;805:422-7.

3. Cibas ES, Ali SZ. The Bethesda System for reporting thyroid cytopathology. Am J Clin Pathol. 2009;132:658-65.

4. Schlumberger MJ, Filetti S, Alexander EK, Hay ID. Nontoxic diffuse goiter, nodular thyroid disorders, and thyroid malignancies. In: Melmed S, Polonsky KS, Larsen PR, Kronenberg HM, editors. Williams Textbook of Endocrinology. $13^{\text {th }}$ ed. Philadelphia: Elsevier Saunders; 2016. p. 449-88.

5. Olson MT, Zelger MA. Thyroid fine-needle aspiration and cytological diagnosis. In: Jameson JL, De Groot LJ, editors. Endocrinology adult and pediatric. $7^{\text {th }}$ edition Philadelphia: Elsevier Saunders; 2016. p. 1417-22.

6. Gharib H, Papini E, Garber JR, Duick DS, Harrell RM, Hegedüs L, et al. American Association of Clinical Endocrinologists, American College of Endocrinology, and Associazione Medici Endocrinologi Medical
Guidelines for Clinical Practice for the Diagnosis and Management of Thyroid Nodules - 2016 Update. Endocr Pract. 2016; 22:622-39.

7. Heller M, Zanocco K, Zydowicz S, Elaraj D, Nayar R, Sturgeon C. Cost-effectiveness analysis of repeat fine-needle aspiration for thyroid biopsies read as atypia of undetermined significance. Surgery. 2012;152:423-30

8. Gabalec F, Cáp J, Ryska A, Vasátko T, Ceeová V. Benign fine-needle aspiration cytology of thyroid nodule: to repeat or not to repeat? Eur J Endocrinol. 2009; 61:933-7.

9. llouz F, Rodien P, Saint-Andre JP, Triau S, Laboureau-Soares S, Dubois $S$, et al. Usefulness of repeated fine-needle cytology in the follow-up of non-operated thyroid nodules. Eur J Endocrinol. 2007;156:303-8.

10. Orlandi A, Puscar A, Capriata E, Fideleff $H$. Repeated fine-needle aspiration of the thyroid in benign nodular thyroid disease: critical evaluation of long-term follow-up. Thyroid. 2005;15:274-8.

11. Merchant $\mathrm{SH}$, Izquierdo R, Khurana KK. Is repeated fine-needle aspiration cytology useful in the management of patients with benign nodular thyroid disease? Thyroid. 2000;10:489-92.

12. Cengic I, Tureli D, Ozden F, Bugdayci O, Aydin H, Aribal E. Thyroid fine needle aspiration biopsy: do nodule volume and cystic degeneration ratio affect specimen adequacy and cytological diagnosis time? Acta Radiol. 2015;56:1203-8. 